MITSUBISHI ELECTRIC RESEARCH LABORATORIES

http://www.merl.com

\title{
Synthetic Aperture Imaging Using a Randomly Steered Spotlight
}

\author{
Liu, D.; Boufounos, P.T.
}

TR2013-070 July 2013

\begin{abstract}
In this paper, we develop a new approach to synthetic aperture imaging inspired by recently developed compressive sensing (CS) methods. Our approach modifies the beam steering pattern of conventional sliding spotlight-mode systems and randomizes it such that with each pulse the beam illuminates a different, randomly chosen, part of the imaged area. The randomization allows the acquisition of the area of interest with a significantly larger effective aperture compared to the conventional sliding spotlight mode and, therefore, with significantly larger resolution. The reconstruction estimates the signal using a model that combines a sparse and a dense component. This model captures the structure of SAR images better than conventional sparse models, typically used in CS, and provides superior reconstruction performance. Our experimental results demonstrate that the proposed randomly steered spotlight array can improve imaging resolution, as measured by the reconstruction SNR and the phase error, without compromising the covered area size.
\end{abstract}

International Geoscience and Remote Sensing Symposium (IGARSS)

This work may not be copied or reproduced in whole or in part for any commercial purpose. Permission to copy in whole or in part without payment of fee is granted for nonprofit educational and research purposes provided that all such whole or partial copies include the following: a notice that such copying is by permission of Mitsubishi Electric Research Laboratories, Inc.; an acknowledgment of the authors and individual contributions to the work; and all applicable portions of the copyright notice. Copying, reproduction, or republishing for any other purpose shall require a license with payment of fee to Mitsubishi Electric Research Laboratories, Inc. All rights reserved. 



\title{
SYNTHETIC APERTURE IMAGING USING A RANDOMLY STEERED SPOTLIGHT
}

\author{
Dehong Liu, Petros T. Boufounos \\ Mitsubishi Electric Research Labs, $\{$ liudh,petros $\}\} @$ merl.com
}

\begin{abstract}
In this paper, we develop a new approach to synthetic aperture imaging inspired by recently developed compressive sensing (CS) methods. Our approach modifies the beam steering pattern of conventional sliding spotlight-mode systems and randomizes it such that with each pulse the beam illuminates a different, randomly chosen, part of the imaged area. The randomization allows the acquisition of the area of interest with a significantly larger effective aperture compared to the conventional sliding spotlight mode and, therefore, with significantly larger resolution. The reconstruction estimates the signal using a model that combines a sparse and a dense component. This model captures the structure of SAR images better than conventional sparse models, typically used in CS, and provides superior reconstruction performance. Our experimental results demonstrate that the proposed randomly steered spotlight array can improve imaging resolution, as measured by the reconstruction SNR and the phase error, without compromising the covered area size.
\end{abstract}

Index Terms - synthetic aperture, random steering, compressive sensing, high resolution

\section{INTRODUCTION}

Synthetic Aperture Radar (SAR) systems exploit the motion of a moving platform to create a large synthetic aperture using a small antenna and, thus, image an area with high resolution. To-date several different modes of SAR operation have been developed, each with different trade-offs with respect to resolution, coverage and implementation complexity.

The most commonly used modes are strip-map and spotlight. In strip-map mode SAR, the radar antenna is pointed towards the same, fixed direction as the platform moves along a path, which is typically linear. In this mode, the radar illuminates a long strip on the ground, covering a large area with relatively low resolution. On the other hand, in spotlight mode SAR, the antenna is steered as the platform moves to always illuminate the same spot on the ground. This mode reduces the area covered, but significantly increases the resolution of the acquired image.

More recently, intermediate modes have been developed, referred to as sliding spotlight SAR or hybrid stripmap-spotlight SAR [1, 2]. These modes explore the trade-off between stripmap and spotlight mode to generate SAR images with improved azimuth resolution compared to the former and improved ground coverage compared to the latter. In the sliding spotlight acquisition mode, the radar antenna is steered such that the beam centers intersect at a point farther away from the radar than the area being illuminated. Depending on the position of this intersection point, this mode approaches either the stripmap or the spotlight mode and, therefore, is a generalization of both. Specifically, as the intersection point moves closer to the imaging area plain, the sliding spotlight mode becomes spotlight mode. If, instead, the intersection point moves farther, towards infinity, the mode approaches the stripmap mode.
In this paper, we use compressive sensing (CS) techniques to significantly improve the resolution provided by the sliding spotlight mode without compromising the size of the imaged area. The mode we develop generalizes the CS-based spotlight mode we developed in earlier work SAR [3]. The resulting system can achieve the same coverage as typical sliding-mode SAR, with significantly improved resolution. The mode we develop exploits a randomly steered antenna array to achieve a larger effective aperture for each point in the imaged area than the typical sliding spotlight. Using CS-based signal models and reconstruction algorithms we can reconstruct the image from the acquired data at much higher resolution, corresponding to the larger effective aperture.

Our work relies heavily in CS principles. Since their introduction such ideas have had significant impact in sensing applications, including radar imaging [4,5]. The main benefit of CS is that it enables robust reconstruction of signals using a smaller number of measurements compared to their Nyquist rate. This sampling rate reduction is achieved using randomized measurements, improved signal models and non-linear reconstruction algorithms. In SAR systems, this translates to significant resolution or coverage improvements. For example, our earlier work on stripmap mode SAR demonstrates that it is possible to significantly increase the azimuth resolution without compromising the range coverage [6]. Similarly, our work on spotlight mode SAR shows that it is possible to significantly increase the area covered, without compromising the resolution [3].

In contrast to our earlier work in [6], which randomizes the timing of the transmitted pulses, the system we develop in this paper operates in the same way as a conventional SAR system: pulses are transmitted and their echoes are received at a uniform pulse repetition frequency (PRF). The resulting system is similar to our spotlight work in [3], which separates the covered area to a small number of spots and randomly steers the beam to cover one of them in each pulse transmission. Each spot is imaged separately and a separate image is reconstructed for each spot.

The present work incorporates more flexible steering. Instead of splitting the scene to a small number of spots and separately imaging each of them, we now treat the scene in it entirety. In particular, at each pulse transmission the beam center is steered randomly such that it covers a spot randomly located uniformly within the imaged area. The imaged area is reconstructed as a whole, properly taking into account the leakage from the sidelobes of each beam. Compared to [3], the resulting system provides improved reconstruction performance. More importantly, it provides the user with greater flexibility: the imaged area may have an arbitrary size, in contrast to [3], in which the size is restricted to integer multiples of the spotlight size.

Our exposition focuses mainly on the CS-based image reconstruction algorithm and principles, assuming uniform random steering of the beam centers. Since SAR images exhibit limited sparsity, our approach is to decompose the underlying SAR image into a sparse and a dense component. We first reconstruct the sparse com- 
(a)

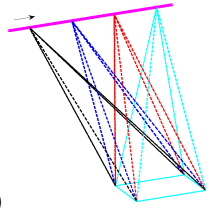

(b)

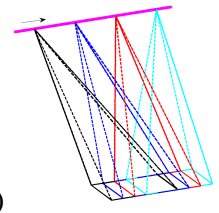

(c)

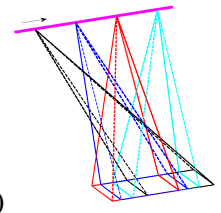

Fig. 1. Pulse beam steering: (a) spotlight mode, (b) sliding spotlight mode, (c) randomly-steered sliding spotlight mode.

ponent using CS-based sparse recovery algorithms. Then we obtain a least-squares estimate of the dense component from the residual data. The sum of the two components is the reconstructed ground reflectivity. Our experiments showed that this hybrid model outperforms CS algorithms that only perform sparse recovery.

The next section describes our proposed steering mode, as well as the computational model for its implementation. Section 3 presents the reconstruction algorithm. In Section 4 we demonstrate the improved performance of our approach compared to classical spotlight-mode SAR using simulation experiments. Section 5 discusses our results and concludes.

\section{SYSTEM MODEL}

In this section, we describe our acquisition model using spotlight mode SAR as a basis. We consider imaging using a linear monostatic uniform virtual array, operating as shown in Fig. 11.a). To image an area or scene, a mobile radar platform moves along a path, transmitting pulses at a uniform rate and recording their echoes from the area of interest. In conventional spotlight mode, the transmitted pulse beam is steered such that its main lobe is always aimed at the same spot of interest.

Each reflection is effectively a convolution of the transmitted pulse with the reflectivity of the illuminated spot. Thus, acquisition can be modeled as a linear operation:

$$
\mathbf{y}=\mathbf{\Phi} \mathbf{x}+\mathbf{n},
$$

where $\mathbf{y}$ denotes the received radar echoes, $\mathbf{x}$ denotes the reflectivity of the imaged area, $\boldsymbol{\Phi}$ models the array acquisition function, and $\mathbf{n}$ is measurement noise.

Image formation aims to determine the signal of interest $\mathbf{x}$ given the array echoes $\mathbf{y}$ and the acquisition function $\boldsymbol{\Phi}$. Least squares solutions, for example, use the inverse or the pseudoinverse of $\Phi$ to determine $\mathbf{x}$ :

$$
\hat{\mathbf{x}}=\boldsymbol{\Phi}^{\dagger} \mathbf{y} \text {. }
$$

For the system to be invertible, the pulse repetition frequency should be sufficiently high given the size of the area imaged.

In the sliding spotlight mode as illustrated in Fig. 1 b), the transmitted pulse beam is steered such that its main lobe aims to a point farther away from the SAR system than the area of interest. Therefore, the spot areas illuminated by each beam are not identical, but sliding in the azimuth direction. This enables imaging of a larger area at the same pulse repetition frequency. The trade-off is that each point in the measured scene is illuminated by a sub-aperture of the whole mobile platform path, thus reducing the available resolution.

Starting with the conventional spotlight mode model in (1), we can derive a simple model from both the sliding spotlight mode and the randomly steered sliding spotlight mode. In this model, we consider the area to be imaged as a whole and denote it using $\mathbf{v}$. At pulse transmission $i$, the beam is steered according to the steering sched- ule, effectively windowing $\mathbf{v}$ through the illumination beampattern. The illuminated area, therefore is a spatially windowed version of $\mathbf{v}$, i.e., $\mathbf{x}_{i}=\mathbf{W}_{i} \mathbf{v}, i=1, \ldots, N$, where $\mathbf{W}_{i}$ denotes the spatial window. Note that $\mathbf{W}_{i}$ can also capture detailed beampattern characteristics, such as the sidelobes.

To model and efficiently implement the system we first presume that the same window $W_{i}$ is used for all pulses, i.e., the system operates in conventional spotlight mode, and use $\mathbf{y}_{i}=\mathbf{\Phi} \mathbf{x}_{i}$ to denote the complete received data, as in (1). However, from all the data in $\mathbf{y}_{i}$ we are only interested in the $i^{t h}$ received echo. Use $\mathbf{E}_{i}$ to denote the operator than only selects that echo, the received echo from the transmission of the $i^{t h}$ pulse is $\mathbf{E}_{i} \boldsymbol{\Phi} \mathbf{W}_{i}$. Thus, the resulting full measurement data, which we denote using $\mathbf{u}$, can be modeled as

$$
\mathbf{u}=\left[\begin{array}{c}
\mathbf{E}_{1} \mathbf{y}_{1} \\
\vdots \\
\mathbf{E}_{N} \mathbf{y}_{N}
\end{array}\right]=\left[\begin{array}{c}
\mathbf{E}_{1} \mathbf{\Phi} \mathbf{W}_{1} \\
\vdots \\
\mathbf{E}_{N} \mathbf{\Phi} \mathbf{W}_{N}
\end{array}\right] \mathbf{v}=\mathbf{\Psi} \mathbf{v}
$$

As described in Sec. 11 our CS-based SAR system uniformly transmits pulses and receives echoes, similarly to conventional SAR systems. The difference is the array steering, as illustrated in Fig. 1 (c). Instead of steering the array to illuminate an adjacent sliding spot as the sensor moves, we illuminate different spots at random locations within the area of interest. Each spot has the same size as the size of a single spot in a conventional spotlight or sliding spotlight SAR system. Both systems are modeled by incorporating the appropriate sliding or randomly steered windows in 3 . While a totally random steering is preferred for robust CS reconstruction, for computational complexity reasons we select each $\mathbf{W}_{i}$ from a discrete set of pre-determined overlapping spatial windows distributed uniformly in the area of interest.

Since each point in the measured scene is illuminated from several positions in the mobile platform path, the effective aperture looking at each point is the whole path of the platform, similar to conventional spotlight mode. In contrast, in sliding spotlight mode, a point is illuminated for only part of the platform path, making the effective aperture, and therefore the resolution, much smaller.

This effective aperture gain has limits. As the scene size increases, all else being equal, each point is illuminated by fewer pulses, i.e. is measured fewer times. Thus, the same number of measurements are used to recover a larger scene. Reconstruction is only possible if the scene is sparser or, in general, exhibits more structure. This exploitable structure finally determines the potential for improvement in resolution over sliding spotlight SAR or in coverage over spotlight SAR.

\section{CS-BASED IMAGE RECONSTRUCTION}

In this section, we focus on the CS-based algorithm to reconstruct the image of the area from the acquired measurements. As we describe above, once the randomized steering of the array beam is determined, the resulting acquisition can be modeled using the linear system in (3). However, in contrast to conventional SAR systems, the linear system described by $\boldsymbol{\Psi}$ is underdetermined. In other words, inverting the system is not straightforward and can only be performed accurately if we have prior information on the signal.

Conventional CS methods use the sparsity of the acquired signal, $\mathbf{v}$, as prior information to regularize the reconstruction. Unfortunately, radar images are not very sparse in the conventional CS sense. While radar images contain strong components in some domain, they also contain a significant residual that sparse methods 
1. Initialize $0<\alpha<1, \widehat{\mathbf{v}}_{s}^{(0)}=\mathbf{0}, \mathbf{u}_{r}^{(0)}=\mathbf{u}$,

2. FOR $k=1: K$

$$
\begin{aligned}
\tilde{\mathbf{v}}^{(k)} & =\boldsymbol{\Psi}^{\dagger} \mathbf{u}_{r}^{(k-1)} \\
\tau^{(k)} & =\max \left(\left|\tilde{\mathbf{v}}^{(k)}\right|\right) \cdot \alpha \\
\mathbf{d}^{(k)} & =\mathcal{H}_{\tau^{(k)}}\left(\tilde{\mathbf{v}}^{(k)}\right) \\
\tilde{\mathbf{u}}^{(k)} & =\boldsymbol{\Psi} \mathbf{d}^{(k)} \\
\beta^{(k)} & =\frac{<\tilde{\mathbf{u}}^{(k)}, \mathbf{u}_{r}^{(k-1)}>}{<\tilde{\mathbf{u}}^{(k)}, \tilde{\mathbf{u}}^{(k)}>} \\
\mathbf{u}_{r}^{(k)} & =\mathbf{u}_{r}^{(k-1)}-\beta^{(k)} \tilde{\mathbf{u}}^{(k)} \\
\widehat{\mathbf{v}}_{s}^{(k)} & =\widehat{\mathbf{v}}_{s}^{(k-1)}+\beta^{(k)} \mathbf{d}^{(k)}
\end{aligned}
$$

END

3. Output

$$
\text { Image: } \widehat{\mathbf{v}}=\widehat{\mathbf{v}}_{s}^{(K)}+\Psi^{\dagger} \mathbf{u}_{r}^{(K)}
$$

Fig. 2. Reconstruction algorithm

cannot easily take into account. For that reason, we propose a CSbased algorithm that, instead of simply treating the image as a sparse signal, decomposes $\mathbf{v}$ into a sparse part $\mathbf{v}_{s}$ and a dense residual $\mathbf{v}_{r}$ :

$$
\mathbf{v}=\mathbf{v}_{s}+\mathbf{v}_{r} .
$$

Thus, substituting (4) into (3), the measured data can also be notionally separated to $\mathbf{u}=\boldsymbol{\Psi}_{\mathbf{v}_{s}}+\boldsymbol{\Psi}_{\mathbf{v}_{r}}$. Treating $\mathbf{u}_{r}=\boldsymbol{\Psi}_{\mathbf{v}_{r}}$ as noise, our approach first computes an estimate of the sparse signal component $\mathbf{v}_{s}$ using standard CS-based methods:

$$
\widehat{\mathbf{v}}_{s}=\arg \min _{\mathbf{v}}\|\mathbf{u}-\mathbf{\Psi} \mathbf{v}\|_{2}^{2} \text { s.t. }\|\mathbf{v}\|_{0}<T .
$$

Using this sparse estimate we can estimate the residual data due to the dense component, i.e., $\widehat{\mathbf{u}}_{r}=\mathbf{u}-\mathbf{\Psi} \widehat{\mathbf{v}}_{s}$. The dense component can then be estimated using least squares:

$$
\widehat{\mathbf{v}}_{r}=\Psi^{\dagger}\left(\mathbf{u}-\boldsymbol{\Psi} \widehat{\mathbf{v}}_{s}\right)
$$

The final image is the sum of the two estimates from (5) and $(6)$.

The algorithm in Fig. 2 efficiently implements this idea. In each iteration the algorithm uses the residual $\mathbf{u}_{r}^{(k-1)}$ to compute an estimate of the so-far unexplained signal $\tilde{\mathbf{v}}^{(k)}$. To obtain the strongest reflectors, a threshold $\tau^{(k)}$ is computed as a fraction of the largest in magnitude signal component. The estimate of the strongest reflectors $\mathbf{d}^{(k)}$ is computed by imposing a hard threshold $\mathcal{H}_{\tau(k)}(\cdot)$ on $\tilde{\mathbf{v}}^{(k)}$, i.e., by setting all components less than $\tau^{(k)}$ in magnitude to zero. This estimate is scaled using $\beta^{(k)}$, such that it explains most of the residual energy in $\mathbf{u}_{r}^{(k-1)}$, and added to the overall signal estimate from the previous iteration $\widehat{\mathbf{v}}_{s}^{(k-1)}$ to produce the current signal estimate $\widehat{\mathbf{v}}_{s}^{(k)}$. The residual $\mathbf{u}_{r}^{(k-1)}$ is updated and the algorithm iterates. After $K$ iterations, the algorithm combines the estimated signal $\widehat{\mathbf{v}}_{s}^{(K)}$ from the $K^{t h}$ iteration and the least squares estimate of the dense component, $\boldsymbol{\Psi} \mathbf{u}_{r}^{(K)}$ to produce the final image $\widehat{\mathbf{v}}$.

Our experiments demonstrated that this reconstruction approach, in which the final image is composed of a sparse and a dense component, outperformed reconstruction approaches using only sparsity regularization. Of course, the dense component can only be estimated subject to the nullspace of the measurement oper- ator $\Psi$. However, this estimate is better than ignoring its presence, typically done in sparse methods. Our experiments also showed that setting $\alpha>0.5$ is preferred for good imaging performance.

In practical SAR systems, the array acquisition function $\boldsymbol{\Phi}$ and its inversion are generally difficult to model accurately and computationally expensive. In our simulation, considering the large squint angle in sliding spotlight arrays, we employ the wave-number algorithm to implement both efficiently [3 7]. The diagonal operators $\mathbf{E}_{i}$ and $\mathbf{W}_{i}$ are straightforward to implement efficiently. The measurement operator $\boldsymbol{\Psi}=\mathbf{E} \boldsymbol{\Phi} \mathbf{W}$ is implemented as a combination of all, as described in (3).

Of course, as the number of possible steering spots increases, this approach to implementation becomes increasingly inefficient; we need to compute one instance of the wave-number algorithm for each possible spot. A more efficient implementation is necessary, but this is not explored in this paper.

\section{EXPERIMENTS}

To verify our approach, we simulated the SAR acquisition followed y reconstruction on an area with complex-valued ground reflectivity using both a sliding spotlight SAR and the proposed randomly steered system. For the simulations we use a typical ground reflectivity image, which contains man-made and natural structures and should be representative of the sparsity of typical SAR images.

In our experiments we discretize the area of interest into a total number of 22 rectangular overlapping spots, sliding in the azimuth direction with a fixed space shift. For the conventional sliding spotlight SAR, we group the whole virtual aperture into 22 sub apertures, correspondingly, with each sub-aperture illuminating one spot sequentially within the area of interest. For the randomly steered spotlight SAR, we pre-design a steering pattern such that each spot is illuminated randomly with uniform probability. Thus, each spot is illuminated by roughly $1 / 22$ of transmitting positions, randomly picked along the virtual aperture.

The result are shown in Fig. 3 From left to right, we plot the true complex-valued ground reflectivity of the area, the reconstructed image using conventional sliding spotlight mode and the reconstructed image using the proposed approach. The top row contains the whole imaged area. To demonstrate the reconstruction detail, we also zoom in two particular regions of the area, shown in the bottom rows.

As evident in the figure-especially when Fig. 3 (f) and (i) is compared with Fig. 3.e) and (h), respectively - the reconstructed image using the randomized acquisition is much sharper compared to the sliding mode acquisition. The fine structures in the ground are significantly less blurred and fine details are discernible. The reason is that, as we describe above, the effective aperture of the randomly steered mode is larger than that of the sliding mode. Thus, using the signal model, our algorithm is able to exploit the structure of the image by identifying the strong reflectors and reconstructing them at much higher resolution. Since these are the dominant components, the resulting image reconstruction performance is improved.

We also plot the point-wise signal-to-noise ratio (SNR) in $\mathrm{dB}$ and phase error in radians of each pixel in the top and bottom rows of Fig. 4. with conventional mode on the left and the proposed mode on the right. Compared to the conventional mode, the proposed sliding mode improves the SNR by $10 \mathrm{~dB}$ and the phase error by more than $70 \%$. More importantly, we observe that the performance is even more improved for strong scattering areas. This is because in every iteration of our reconstruction algorithm, we always try to estimate the strongest scattering pixels accurately as first priority, leaving the 


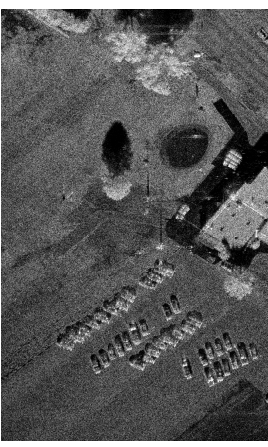

(a)

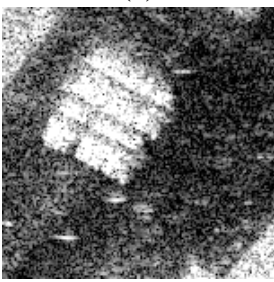

(d)

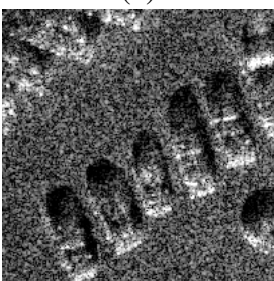

(g)

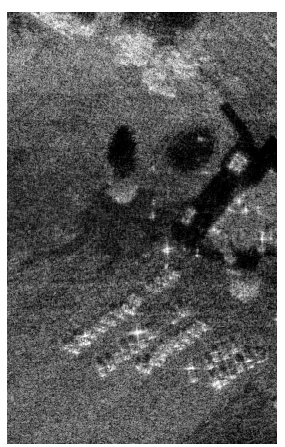

(b)

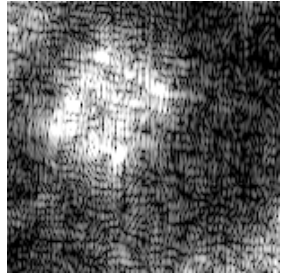

(e)

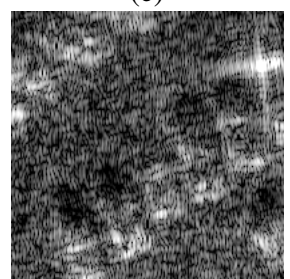

(h)

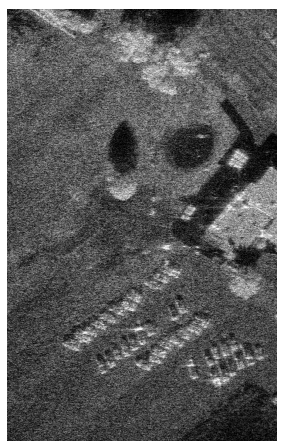

(c)

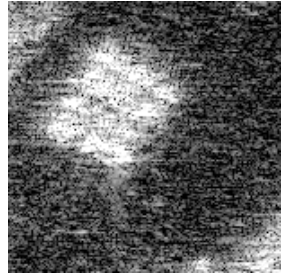

(f)

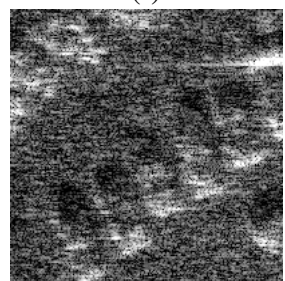

(i)
Fig. 3. (a) True ground reflectivity, (b) conventional sliding spotlight imaging result, and (c) CS based sliding spotlight imaging result.(d)and (g) are zoomed in areas of (a); (e) and (h) are zoomed in areas of (b); and (f) and (i) are zoomed in areas of (c).

weaker ones for a secondary estimate. The small phase error, especially at strong reflectors, is beneficial for future image analysis such as object detection and 3-D imaging where the phase information is important.

\section{CONCLUSION}

As our experiments demonstrate, there is great potential for improvement in SAR systems using CS-based methods. However, one should be careful in implementing those, as the standard sparsity assumptions do not immediately apply. Our work shows that randomizing the steering of the array and exploiting the partial sparsity of the image can significantly improve the performance of sliding spotlight systems, without compromise in the covered area.

While our model has demonstrated those improvements, we believe there is significant scope for better models of the structure in SAR images. These models will enable further advances in reconstruction performance and signal quality. There is also significant scope for improvement in the complexity of computing the forward and the adjoint operators derived by random steering. We think both these research directions will enhance SAR performance and capabilities.

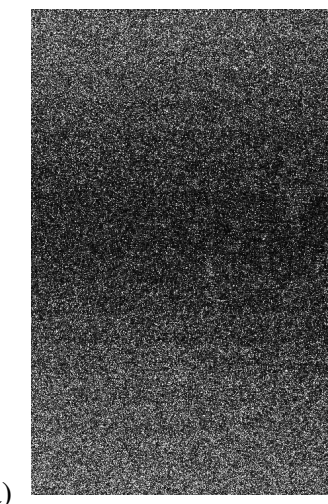

(b)

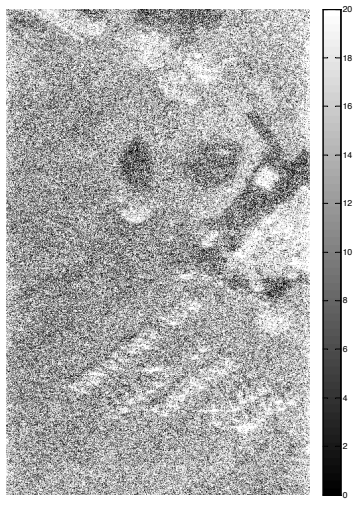

(c)

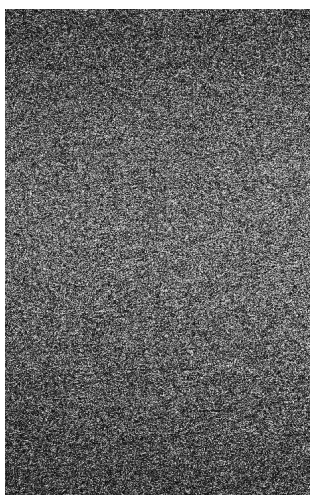

(d)

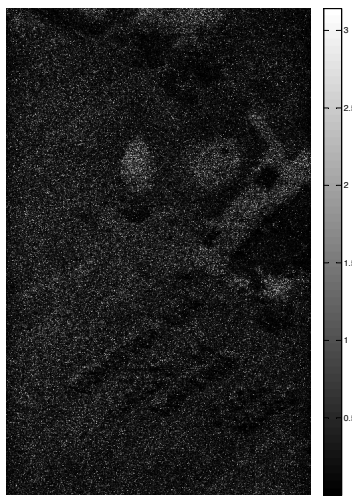

Fig. 4. Magnitude SNR (dB) of (a) conventional sliding spotlight SAR and (b) randomly steered spotlight SAR. Phase error (radians) of (c) conventional sliding spotlight SAR and (d) randomly steered spotlight SAR.

\section{REFERENCES}

[1] G. Franceschetti, R. Guida, A. Iodice, D. Riccio, and G. Ruello, "Efficient simulation of hybrid stripmap/spotlight SAR raw signals from extended scenes," IEEE Trans. Geoscience and Remote Sensing, vol. 42(11), pp. 2385-2395, Nov 2004.

[2] J. Mittermayer, R. Lord, and E. Börner, "Sliding spotlight SAR processing for TerraSAR-X using a new formulation of the extended chirp scaling algorithm," in IEEE International Geoscience and Remote Sensing Symposium (IGARSS), 2003.

[3] D. Liu and P. T. Boufounos, "Random steerable arrays for synthetic aperture imaging," in IEEE International conference on Acoustics Speech and Signal Processing(ICASSP), 2013.

[4] R. Baraniuk and P. Steeghs, "Compressive radar imaging," in IEEE Radar Conference, MA, April 2007.

[5] L. C. Potter, E. Ertin, J. T. Parker, and M. Cetin, "Sparsity and compressed sensing in radar imaging," Processings of the IEEE, vol. 98, pp. 1006-1020, June 2010.

[6] D. Liu and P. T. Boufounos, "High resolution SAR imaging using random pulse timing," in IEEE International Geoscience and Remote Sensing Symposium (IGARSS), 2011.

[7] I. G. Cumming and F. H. Wong, Digital processing of synthetic aperture radar data: algorithms and implementation, Artech House, 2005. 